Published in final edited form as:

Curr Cardiol Rep. ; 21(12): 152. doi:10.1007/s11886-019-1238-1.

\title{
Cardiac Sarcoidosis
}

Pranav Mankad ${ }^{1}$, Brian Mitchell ${ }^{1}$, David Birnie ${ }^{2}$, Jordana Kron ${ }^{1}$

${ }^{1}$ Pauley Heart Center, Virginia Commonwealth University Medical Center, P.O. Box 980053, Richmond, VA 23298-0053, USA

${ }^{2}$ University of Ottawa Heart Institute, Ottawa, Canada

\section{Abstract}

Purpose of Review-In this state-of-the-art review, we highlight our current understanding of diagnosis, assessment, and management of cardiac sarcoidosis (CS), focusing on recently published data and expert consensus statement guidelines.

Recent Findings-Academic interest in cardiac sarcoidosis research has increased over the past decade along with increased clinical awareness among clinicians. In 2014, the Heart Rhythm Society published the first expert consensus statement on diagnosing and managing arrhythmias associated with CS. Cardiac magnetic resonance has emerged as a valuable tool both for diagnosing CS and predicting risk of life-threatening ventricular arrhythmias based on burden of late gadolinium enhancement. Cardiac fluorodeoxyglucose-positron emission tomography now plays a role in diagnosis, risk stratification, and assessing response to immunosuppressive therapy.

Summary-Collaborative, multidisciplinary research efforts are needed to further our understanding of this rare, complex disease. Two large multicenter prospective registries-the international Cardiac Sarcoidosis Consortium and the Canadian Cardiac Sarcoidosis Research Group - are enrolling patients to help provide insights into the natural history of the disease and current treatment strategies. Future research should focus on randomized controlled trials comparing different treatment strategies and identifying and testing novel therapeutic agents.

\section{Keywords}

Cardiac sarcoidosis; Cardiac magnetic resonance; Positron emission tomography; Implantable cardioverter defibrillator; Sudden cardiac death

\section{Introduction}

Sarcoidosis is a systemic inflammatory disease defined histologically by the formation of non-caseating granulomas and subsequent tissue scarring. Sarcoidosis can involve virtually any organ system, most commonly lungs, liver, skin, gastro-intestinal system, eyes, and

\footnotetext{
Jordana Kron, jordana.kron@vcuhealth.org.

Conflict of Interest Pranav Mankad, Brian Mitchell, David Birnie, and Jordana Kron declare that they have no conflict of interest.

Human and Animal Rights and Informed Consent This article does not contain any studies with human or animal subjects performed by any of the authors.

Publisher's Note Springer Nature remains neutral with regard to jurisdictional claims in published maps and institutional affiliations.
} 
neurologic system [1]. The prevalence of sarcoidosis throughout the world ranges from 4.7 to 64 in 100,000 and is highest in northern Europeans and African Americans and more common in women than men. The annual incidence of sarcoidosis in the USA has been estimated at 10.9 per 100,000 in whites and 35.5 per 100,000 in African Americans [2••].

Autopsy data show up to $25 \%$ of patients with systemic sarcoidosis have cardiac involvement [3]. Cardiac involvement in sarcoidosis portends a worse prognosis [4]. Cardiac manifestations depend on the extent and location of the heart involved and can range from asymptomatic presentation to ventricular tachycardia (VT), high-grade atrioventricular (AV) block, or heart failure. Because sudden cardiac death can be the first presentation of cardiac sarcoidosis (CS) due to brady- or tachyarrhythmias, increased awareness and understanding of this disease process is essential. The diagnosis of CS has increased significantly over the past 25 years, likely attributed to improved imaging techniques, standardized diagnostic algorithms, and enhanced awareness of the disease process. Despite increased prevalence over the recent years, given the rarity of disease, the majority of data is still based on nonrandomized trials and expert opinions. More recent data provides additional support for the 2014 HRS Expert Consensus Statement.

\section{Diagnosis: Current Practice, Emerging Tools, and Evidence Criteria Updates}

Three major guidelines exist for the diagnosis of CS: the 2014 Heart Rhythm Society (HRS) document, the World Association of Sarcoidosis and Other Granulomatous Disorders Sarcoidosis Organ (WASOG) criteria, and the Japanese Ministry of Health \& Welfare (JMHW) guidelines with updates by the joint committee of the Japanese Society of Sarcoidosis and Other Granulomatous Disorders and the Japanese College of Cardiology in 2017. The WASOG criteria were devised in 1999 as A Case Control Etiology of Sarcoidosis Study (ACCESS) instrument in 1999 and were revised by WASOG in 2014. The WASOG criteria were expanded upon by the HRS consensus statement [2••], with updates to the criterion for clinical diagnosis. The HRS diagnostic criteria include two diagnostic pathways: (1) histologic diagnosis from myocardial tissue or (2) clinical diagnosis from invasive and non-invasive studies (Table 1). The clinical diagnostic pathway requires histological diagnosis of extracardiac sarcoidosis and one or more clinical or imaging criteria. Using the HRS criteria, isolated CS can thus only be diagnosed with myocardial tissue, making this challenging diagnosis difficult to confirm. In 2017, JMHW guidelines were revised and several significant changes were made to the criteria: (1) guidelines now allow for diagnosis of isolated CS without a positive endomyocardial biopsy, (2) fatal ventricular arrhythmia (sustained ventricular tachycardia and ventricular fibrillation) was added to the major criteria alongside high-grade atrioventricular block, (3) abnormal ventricular wall anatomy was moved from the minor to major criteria, (4) elevated myocardial uptake with FDG-PET was moved to the major criteria, and (5) late gadoliniumenhanced MRI was moved from the minor to major criteria [5••]. Table 1 shows the HRS and JMHW Diagnostic Criteria. 


\section{Imaging Modalities}

Echocardiogram-Echocardiogram is not a sensitive tool for CS diagnosis compared with CMR or PET (Fig. 1). However, transthoracic echocardiogram (TTE) is specific and abnormalities can be useful in identifying cardiac dysfunction in biopsy-proven extracardiac sarcoidosis, leading to further imaging to assess for CS. In a retrospective study of patients with biopsy-proven CS, when combined with symptoms and/or Holter monitor findings, TTE had a sensitivity ranging from 71 to $84 \%$. This increase in sensitivity was attributable to the addition of symptoms and/or Holter monitor findings, and thus by itself, TTE does not improve the sensitivity of CS recognition [6]. Because left ventricular ejection fraction (LVEF) is one of the strongest prognostic indicators in CS, once the diagnosis is made, this relatively inexpensive imaging modality has a role in the management of CS patients.

Cardiac Magnetic Resonance-Cardiac magnetic resonance (CMR) is a valuable tool for diagnosis of CS primarily by identifying late gadolinium enhancement (LGE) in the myocardium with a characteristic pattern of patchy and multifocal uptake with sparing of the endocardial border. It is a very sensitive and specific test with both ranging in high $90 \mathrm{~s}$ in some studies (Fig. 1). An association between late gadolinium enhancement and poor prognosis has been shown in a number of studies. A meta-analysis comprising 7 studies and 694 subjects suggested that positive LGE among CS patients was associated with increased risk of cardiovascular death or ventricular arrhythmia [7]. Two hundred and five patients with a diagnosis of extra-cardiac sarcoidosis and left ventricular ejection fraction (LVEF) > $50 \%$ were risk stratified by assessing LGE burden using CMR, with 41 (20\%) having positive LGE. Twelve of 205 patients died or had VT during a mean follow-up time of 36 months, and 10 of these $12(83 \%)$ were LGE positive-representing a greater than 20 times higher rate of death or VT per year compared with the LGE-negative group [8]. A metaanalysis of ten studies involving 760 total patients showed that patients with LGE had higher odds for all-cause mortality (odds ratio, 3.1) and greater odds of arrhythmogenic events (ventricular arrhythmia, ICD shock, sudden cardiac death) plus all-cause mortality (odds ratio, 10.7) compared with patients without LGE [9]. Figure 2 shows 3 T CMR images of a patient with cardiac sarcoidosis demonstrating patchy LGE involving the base of the anteroseptum and multiple areas of the anteroseptum and inferoseptum.

While useful and sensitive for diagnosis of CS, CMR has its limitations. CMR with LGE is less sensitive regarding active myocardial inflammation and thus is not currently useful in guiding immunosuppressive therapy. However, novel CMR techniques utilizing T1 and T2 mapping are being evaluated, and multiple studies suggest this may allow detection of myocardial inflammation. Compared with control subjects, patients with biopsy-proven extra-cardiac sarcoidosis had significantly higher $\mathrm{T} 1$ and $\mathrm{T} 2$ indices and that myocardial native T1 and T2 had higher discriminatory accuracy compared with HRS and JMHW guidelines. Further, T1 and T2 indices were significantly reduced among patients undergoing anti-inflammatory therapy, suggesting a role in monitoring response to treatment [10]. 
FDG-PET Imaging - 18F-fluorodeoxyglucose positron emission tomography (FDG-PET) is most useful for imaging areas of myocardial inflammation and in measuring response to immunosuppressive therapy. FDG-PET shows very good sensitivity and specificity for diagnosis of CS. A meta-analysis analyzed 18 FDG-PET studies in 891 patients and found a pooled sensitivity of FDG-PET for diagnosis of CS of 0.84 (95\% CI 0.71 to 0.91 ) and a pooled specificity of 0.83 (95\% CI 0.74 to 0.89 ). While pooled sensitivity and specificity were higher in studies that also assessed MPI, these were not statistically significant. However, the diagnostic odds ratio for CS using FDG-PET (14.2) was improved when MPI was utilized (25.7) [11].

The presence of FDG uptake and perfusion defects on PET is associated with higher risk of death or VT. Specifically, patients with focal RV inflammation had a 5-fold higher event rate compared with patients with normal perfusion and metabolism, suggesting focal RV involvement could be a marker for more severe disease [12]. A retrospective study of 203 patients referred for FDG-PET for evaluation of CS found that quantitative scores of the extent and severity of perfusion metabolism mismatch and of variation in FDG uptake provided incrementally superior prognostic information compared with other qualitative methods. The most robust method was the summed rest score (SRS) of all segments with perfusion metabolism mismatch, followed by a second score measuring the coefficient of variation of the FDG signal representing metabolic heterogeneity [13].

In addition to aiding with diagnosis and prognosis, FDG-PET can help to monitor response to therapy. Frequency of follow-up has not been defined, but we typically repeat FDG-PET every 6-9 months to reevaluate degree of inflammation. Figure 3 shows FDG-PET before and after therapy with methotrexate in a 63-year-old woman with CS and complete heart block, demonstrating a significant decrease in FDG uptake suggesting adequate response to therapy.

Combined Use of FDG-PET and CMR-Despite the respective limitations of FDGPET and CMR imaging, the combination of both appears to have a complimentary effect on strengthening the diagnostic likelihood of CS. A 2018 analysis reported on the diagnostic benefits of combining CMR and FDG-PET for the diagnosis of CS. The study retrospectively reinterpreted both CMR and FDG-PET scans in a cohort of 107 patients, and blinded readers assigned a likelihood of CS—no CS, possible CS, probable CS, or highly probable CS—based on predefined criteria. Overall, 48 (45\%) patients were reclassified (compared with single modality imaging), with 32 of 48 being reclassified to higher overall likelihood and $11 \%$ were reclassified as having a highly probable likelihood of CS.

Endomyocardial biopsy was performed on 38 patients (35\%), with 21 of these diagnosed as having highly probably CS — with both abnormal FDG on PET and LGE on MRI-however, only 3 had positive biopsies [14]. In their joint procedural position statement, Slart et al. recommend addition of FDG-PET to increase diagnostic accuracy and confidence in setting of normal or equivocal CMR despite high suspicion for disease [15]. Another study found that the sensitivity for detecting CS was 0.85 for PET and 0.82 for CMR, but that hybrid PET/CMR was superior with a sensitivity of 0.94 [16]. 
Biomarkers-At this time, there are no biomarkers available to reliably identify and monitor cardiac sarcoidosis activity. A 2018 study of 172 patients with diagnosed sarcoidosis compared biomarkers between non-CS and CS patients, and found that BNP was higher in the CS group, that BNP identified CS with a sensitivity of $85 \%$, and that BNP was a predictor of heart failure among CS patients, with a hazard ratio of 7.84 [17]. Given their contribution to the development of several cardiovascular diseases, micro-RNA has been identified as a possible marker for CS, with one study indicating that serum miR-126 and miR-223 could be potential diagnostic biomarkers for CS [18].

Using TriNetX, a global health research network able to perform real-time analyses on electronic medical records of $>22$ million patients from 19 health care organizations predominantly in the USA, we analyzed C-reactive protein (CRP) levels in 31,421 sarcoidosis patients [19]. We identified 2166 sarcoidosis patients with VT, CHB, HF, or arthritis. Compared with a baseline cohort of patients with sarcoid arthritis without symptomatic cardiac involvement, we found that CRP is elevated in patients with sarcoidosis and VT or heart failure compared with patients without active cardiac sarcoidosis. The mean CRP in patients with VT was significantly higher than the control group without VT (5.79 \pm 6.94 vs $4.13 \pm 5.73, p=0.0005)$. CRP was also elevated in patients who had sarcoidosis with HF compared with patients without HF $(5.66 \pm 6.66$ vs $3.88 \pm 5.48, \mathrm{p}<0.0001)$. There was no significant difference in mean CRP in sarcoidosis with CHB compared with control $(4.67 \pm 6.81$ vs $4.18 \pm 5.85, \mathrm{p}=0.50)$. This data suggests that innate immunity may play an important role in cardiac sarcoidosis and could potentially be a diagnostic or therapeutic target going forward.

Endomyocardial Biopsy-EMB is a valuable means to obtain a histologic diagnosis of CS; however, EMB is limited by low sensitivity, high false negative rate, and complications associated with tissues sampling including arrhythmias, conduction abnormalities, and risk of cardiac tamponade. EMB is typically performed via fluoroscopic or echocardiographic guidance. A study examining the efficacy of electrogram-guided EMB found that in patients with suspected myocarditis or CS, abnormal or low-amplitude electrograms increased the diagnostic yield for abnormal histopathological findings [20]. The HRS Consensus Statement recommends that physicians consider using electro-anatomic map or image guidance for EMB [2••].

\section{Management: Current Practice, Emerging Therapies, and Evidence Immunosuppression}

Despite lack of survival benefit, corticosteroids are the mainstay of treatment, while other immunomodulators are second line for patients' refractory to corticosteroids. While some studies show improvement in or suppression of arrhythmias after steroid therapy, data is conflicting. A retrospective study assessing outcomes in 68 patients after initiation of steroid therapy found that 20 of 68 (29\%) experienced VTs, and among those, 14 (70\%) experienced VTs within the first 12 months after initiation of steroid therapy [21].

Steroid-sparing agents are utilized to minimize the adverse side effect of steroids given the typically prolonged duration of therapy for patients with CS. These medications include 
disease-modifying anti-rheumatic drugs like methotrexate and tumor necrosis factor alpha inhibitors like infliximab [22]. Suggested algorithms propose addition of a second line, steroid-sparing agent in situations where the disease relapse or lack of improvement noted while on corticosteroid therapy. Azathioprine and cyclophosphamide have also been used in refractory cases [23].

Data regarding head to head comparison of corticosteroids and immunosuppressants are sparse. One prospective open-label study compared outcomes in CS patients receiving a combination therapy of low-dose steroids and weekly methotrexate versus steroids alone. The authors found that at 3 years, LVEF, cardiothoracic ratio, and NT-proBNP levels were stabilized in the combination therapy group, but not the steroids alone group [22]. Data from the Cardiac Sarcoidosis Consortium, an international multicenter CS registry, found that of 318 CS patients, 84 (26.4\%) were on steroids alone, 72 (22.6\%) were on steroids and a steroid-sparing agent, and $31(9.7 \%)$ were on steroid-sparing agent alone. Methotrexate was the most commonly used steroid-sparing agent, but it should be noted that the centers in the Consortium are predominantly high-volume sarcoidosis referral centers [24].

\section{Conduction Abnormalities}

Although cardiac sarcoidosis can affect any part of the conduction system, resulting in RBBB, LBBB, AV block of any degree, and sinus node arrest, RBBB and AV block are much more common. AV block is a major complication of CS, due to the predilection for CS inflammation to affect the basal interventricular septum and thus the conduction system. All patients < 60 years of age who present with high-degree AV block should be screened for CS. The 2014 HRS expert consensus statement on arrhythmias associated with CS suggested an algorithm for patients with unexplained Mobitz II or third-degree AV block who are younger than 60 years. This includes initial chest CT and/or advanced cardiac imaging with CMR or FDG-PET, with reflex to biopsy for confirmation if imaging is positive [2••].

Corticosteroids have been the mainstay of treatment for immunosuppression over the years. A meta-analysis showed that $47 \%$ of patients treated with corticosteroids had AV recovery compared with $0 \%$ of those who were not [25].

\section{Ventricular Arrhythmias}

Antiarrhythmics have not been studied in randomized trials in this population. It is our practice to commonly use sotalol and amiodarone for ventricular arrhythmias, although amiodarone should be used with caution given the common involvement of the lungs and liver in sarcoidosis patients. Class IC antiarrhythmics including flecainide and propafenone should be avoided in CS patients [2••]. Catheter ablation can be useful to suppress arrhythmic burden or to prevent recurrence of ventricular arrhythmia; however, the rates reported vary for different endpoints and range from 25 to $100 \%$ [26, 27].

The role of corticosteroids on ventricular arrhythmia outcomes remains unclear [21]. In CS patients with frequent symptomatic VT and evidence of myocardial inflammation, immunosuppression in combination with antiarrhythmic medications can be useful to reduce VA burden [28]. A combined treatment approach with steroids and antiarrhythmic medications, followed by radiofrequency catheter ablation if needed, was successful in $63 \%$ 
of patients in one study [29]. The efficacy of catheter ablation for VT in CS depends on the defined endpoint. In a study by Muser et al., catheter ablation in VT patients with CS resulted in long-term arrhythmia-free survival in $40 \%$ of the patients; however, when evaluating significant reduction in arrhythmic burden, up to $90 \%$ patients had positive outcome [30]. Yalagudri et al. demonstrated that tailoring approach to management of VT in CS patients to the phase of disease can improve clinical outcomes. Eighteen patients with unexplained VT after ruling out other causes of VT (including scar from ischemic cardiomyopathy, outflow tract VT, fascicular VT) underwent FDG-PET CT scan. If there was increased FDG uptake in myocardium, they were classified as VT due to inflammation $(\mathrm{N}=14)$. These patients were treated with ICD, immunosuppression, and antiarrhythmics. If they had recurrence and on repeat FDG-PET CT scan had increased myocardial FDG uptake, their immunosuppression was intensified. Patients who did not have myocardial FDG uptake on initial PET CT scan $(\mathrm{N}=4)$ were treated with antiarrhythmics and ICD; if they did not have good clinical response then underwent catheter ablation. Thirteen of the 14 patients in the myocardial inflammation cohort remained free of VT at their last follow-up visit (mean 38.2 months). In the VT due to myocardial scar cohort, all four patients had no FDG uptake on initial PET CT and underwent ICD and antiarrhythmic drug therapy; however, all patients had VT recurrence and underwent catheter ablation [31•].

Even though studies have demonstrated variable efficacy for catheter ablations in management of VT in CS patients, immunosuppression likely has a role if there is evidence of active inflammation. The data from observational non-controlled case series support the use of catheter ablation for refractory VT in CS patients. When reduction of arrhythmia burden is defined as an endpoint, the success rate of ablation is observed to be around $88 \%$ with negligible increase in major complication rates from the procedure [27]. If there is no active inflammation in the myocardium, ablation should have an earlier role in the treatment algorithm.

\section{Risk Stratification for Sudden Cardiac Death}

Identifying CS patients at risk for SCD remains a clinical challenge. CS patients who have had SCD or sustained VT/VF have a high risk of recurrent events and a class I indication for ICD [2••]. Left ventricular ejection fraction (LVEF) $<35 \%$ is also associated with high rates of ventricular arrhythmias and appropriate ICD therapies [32-34]. In a multicenter retrospective study of $235 \mathrm{CS}$ patients, patients who received appropriate ICD therapy had a lower mean LVEF (38.1\%) compared with those who did not receive an appropriate therapy $(48.5 \%)$. In addition, the mean LVEF was lower in patients who received $\geq 5$ appropriate ICD therapies $(35.9 \pm 15.5)$ compared with patients who received $<5$ appropriate ICD therapies $(47.3 \pm 15.0)$ [32].

The 2014 HRS Expert Consensus Statement recommends ICD implant for CS patients with an indication for permanent pacemaker implantation [2••]. This recommendation is supported by recently published Finnish data from the MIDFIN database evaluating risk of sudden death and ventricular arrhythmias in CS patients with AV block. While risk of SCD and VA was highest in patients with prior VT or severe LV dysfunction with EF < 35\%, CS patients with lone AV block and normal LV function had a $9 \%$ risk of SCD and $24 \%$ risk of 
SCD/VT at 5 years [35•]. The 2018 ACC/AHA/HRS Guideline on the Evaluation and Management of Patients with Bradycardia and Cardiac Conduction Delay agreed with the recommendation to implant an ICD in CS patients with an indication for pacing. In CS patients with second- or third-degree AV block who are on chronic stable doses of antiarrhythmic or beta blocker therapy, the experts feel it is reasonable to proceed to permanent pacing without a drug washout period [36].

Programmed electrical stimulation has been used as a way to identify patients at risk for VT and SCD. However, it remains unclear if it is more predictive than LVEF as the inducibility of sustained ventricular arrhythmias inversely correlates with LVEF [37]. Recent data evaluated the usefulness of risk stratification with EPS, electrophysiology study in CS patients with preserved LV and RV function and found that $6 \%$ of patients (7 out of 120) had inducible VT on EPS and received ICD. Three of these patients (43\%) later received ICD therapies for VAs. This data suggests that EPS can be a useful tool in risk stratifying patients with CS with normal LV and RV function as positive EPS can predict future VAs to a certain degree [38].

Multiple studies have demonstrated that late gadolinium enhancement is associated with increased all-cause mortality and increased ventricular arrhythmias when compared with those without LGE [7-9]. Quantity of LGE also appears to be related to the risk of future SCD, for example, in one study, absence of > 8\% LGE of LV myocardium had a 95\% negative predictive value [39]. The presence of FDG uptake and perfusion defects on PET is associated with higher risk of death or VT [12]. RV involvement either noted via LGE on CMR or FDG-PET strongly predicts ventricular arrhythmias in patients with CS and is an independent predictor of SCD or VT [12].

\section{Indications for ICD Implantation}

In patients with cardiac sarcoidosis and sustained VT or have survived sudden cardiac arrest or have LVEF $35 \%$ or less despite optimal medical therapy and a period of immunosuppression in the presence of active inflammation, ICD is a class 1 recommendation if expected survival is greater than 1 year [2••]. In patients with CS and LVEF greater than $35 \%$ with syncope and/or myocardial scar by CMR and/or inducible sustained ventricular arrhythmia on EPS and/or have indication for permanent pacing, ICD

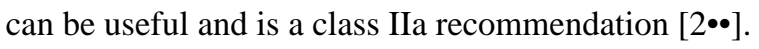

One important recommendation consistent across the guidelines is that CS patients who require permanent pacing for AV block benefit from ICD implantation. The 2014 HRS Expert Consensus Statement and 2017 AHA/ACC/HRS Guideline for Management of Patients with Ventricular Arrhythmias and the Prevention of SCD both recommend ICD implantation if there is an indication for permanent pacemaker implantation (class IIa). More recent data by Nordenswan et al. showed that AVB in the setting of CS is not a benign entity. In patients with $\mathrm{AVB}$ and preserved $\mathrm{EF}(>50 \%)$, the rate of SCD was $9 \%$ at 5 years and in patients with AVB and mildly reduced EF (35-50\%), the rate of SCD was noted to be $14 \%$ at 5 years $\left[35^{\bullet}\right]$. 
A major difference between the HRS 2014 and 2017 Ventricular Arrhythmia guidelines is that the 2017 AHA/ACC/HRS guideline recommends ICD implantation in patients with LVEF 35\% or greater and concomitant evidence of "extensive" myocardial scar by CMR or PET. Importantly, how much LGE constitutes "extensive" scar was not defined [28]. In contrast, for patients with normal LVEF, the 2014 consensus statement recommends considering CMR and if positive, proceeding with EPS. If EPS is positive, ICD implantation is recommended $[2 \bullet \bullet]$.

\section{Isolated Cardiac Sarcoidosis}

As the diagnosis of cardiac sarcoidosis has increased over the years due to increased awareness of the disease and improved diagnostic criteria, the phenomenon of isolated cardiac sarcoidosis has been more and more recognized. Japanese CS guidelines in 2017 were the first set of guidelines that incorporated criteria to diagnose isolated cardiac sarcoidosis. Isolated cardiac sarcoidosis requires diagnosis of CS and absence of sarcoid involvement of any other organ [5*•]. The prevalence of isolated cardiac sarcoidosis varies and is reported between 27 and 54\% in different studies [40]. However, a recent prospective single-center study assessed the rate of isolated CS in patients who presented with clinical manifestation of CS using whole body FDG-PET-CT. The study used a very similar definition of isolated CS as the 2017 Japanese guideline. The study found that only $1 / 31$ patient $(3.2 \%)$ had isolated CS. This is likely due to the highly sensitive diagnostic method used in this study at picking up sarcoid involvement of other organs, including lymph nodes. Adamson described two patients who were initially diagnosed with isolated CS, who went on to develop extra-cardiac disease [41]. These results indicate difficulty in assessing prevalence of isolated cardiac sarcoidosis. Further research is needed to determine if isolated cardiac sarcoidosis is prognostically different than systemic sarcoidosis with cardiac involvement. A high index of suspicion is needed to make this challenging diagnosis.

\section{Future Research Imperatives}

Awareness of cardiac sarcoidosis has increased significantly in the past decade. In 2014, HRS published the first guidelines to focus on arrhythmia diagnosis and management in this challenging patient population [2••]. Many important questions remain unanswered regarding the natural history of CS; optimal diagnostic strategy; most effective treatment including monitoring and duration; and ideal risk stratification for sudden death.

Highlighting the dearth of high-quality data in this burgeoning field, none of the diagnostic schemes has been prospectively studied or validated and no therapies have been compared in a randomized controlled trial.

Several important endeavors are underway that we hope will lead to a seismic shift in the understanding of cardiac sarcoidosis in our lifetime. The Cardiac Sarcoidosis Consortium, an international prospective registry, was created in 2011 and currently has more than 500 patients enrolled from more than 20 centers. The Canadian Cardiac Sarcoidosis Research Group has a multicenter prospective cohort (CHASM-CS) with 1500 participants (NCT01477359). Collaborative research groups such as these are key to moving the field 
forward, but registry data have limitations of missing data and heterogeneity of patients and treatment regimens.

The CHASM-CS RCT (NCT03593759) is the first randomized controlled treatment study of CS, comparing initial treatment of symptomatic CS with prednisone alone vs prednisone with a rapid taper plus methotrexate for 6 months. This ground-breaking trial has begun enrollment. Along with the primary endpoint of summed perfusion rest score on FDG-PET, the study will evaluate multiple secondary endpoints including mortality, cardiovascular hospitalization, quality of life scores, arrhythmia burden, and biomarkers. In addition to leveraging international collaborations to perform head to head comparisons of current therapies for CS, development and identification of novel therapies are also needed. The MRI Assessment of Granulomas in Cardiac Sarcoidosis - Anakinra Randomized Trial (MAGiC-ART) (NCT04017936), a randomized controlled trial which will evaluate treatment of CS with the IL-1 blocker anakinra, is in the pre-enrollment phase.

\section{Conclusion}

Cardiac sarcoidosis occurs in up to $25 \%$ of patients with sarcoidosis in other organs and can cause conduction abnormalities, tachyarrhythmias, sudden death, and heart failure.

Diagnosis of cardiac sarcoidosis in the absence of systemic involvement remains challenging. Increased awareness of CS and an effort to simplify diagnostic criteria has led to increased diagnosis over the last several years. There is increasing evidence that CMR and FDG-PET can provide prognostic data in CS patients. Increasing use of novel imaging modalities like T1 and T2 CMR mapping to differentiate fibrosis from active inflammation can potentially have an important role in diagnostic and management algorithm in future if studies validate these techniques. Although prognosis of cardiac sarcoidosis has improved over the years, disease-modifying therapies with fewer side effects and further understanding and targeting the role of immune system still remain important areas of research. Multicenter research to accelerate understanding of the disease process is key going forward.

\section{References}

Papers of particular interest, published recently, have been highlighted as:

- Of importance

•• Of major importance

1. Valeyre D, Prasse A, Nunes H, Uzunhan Y, Brillet P-Y, Müller-Quernheim J. Sarcoidosis. Lancet 2014;383:1155-67. [PubMed: 24090799]

2••. Birnie DH, Sauer WH, Bogun F, et al. HRS expert consensus statement on the diagnosis and management of arrhythmias associated with cardiac sarcoidosis. Heart Rhythm. 2014;11:1304 23First published expert consensus statement on the diagnosis and management of arrhythmias in cardiac sarcoidosis. Includes guidelines on screening and diagnosis of cardiac sarcoidosis.

3. Silverman KJ, Hutchins GM, Bulkley BH. Cardiac sarcoid: a clinicopathologic study of 84 unselected patients with systemic sarcoidosis. Circulation. 1978;58:1204-11. [PubMed: 709777]

4. Kim JS, Judson MA, Donnino R, Gold M, Cooper LT, Prystowsky EN, et al. Cardiac sarcoidosis. Am Heart J. 2009;157:9-21. [PubMed: 19081391] 
5••. Terasaki F, Yoshinaga K. New guidelines for diagnosis of cardiac sarcoidosis in Japan. Ann Nucl Cardiol. 2017;3:42-5First set of guidelines that include criteria to diagnose isolated cardiac sarcoidosis.

6. Kouranos V, Tzelepis GE, Rapti A, et al. Complementary role of CMR to conventional screening in the diagnosis and prognosis of cardiac sarcoidosis. JACC Cardiovasc Imaging. 2017; 10:1437-47. [PubMed: 28330653]

7. Hulten E, Agarwal V, Cahill M, et al. Presence of late gadolinium enhancement by cardiac magnetic resonance among patients with suspected cardiac sarcoidosis is associated with adverse cardiovascular prognosis: a systematic review and meta-analysis. Circ Cardiovasc Imaging. 2016;9:1-9.

8. Murtagh G, Laffin LJ, Beshai JF, et al. Prognosis of myocardial damage in sarcoidosis patients with preserved left ventricular ejection fraction: risk stratification using cardiovascular magnetic resonance. Circ Cardiovasc Imaging. 2016 10.1161/CIRCIMAGING.115.003738.

9. Coleman GC, Shaw PW, Balfour PC, Gonzalez JA, Kramer CM, Patel AR, et al. Prognostic value of myocardial scarring on CMR in patients with cardiac sarcoidosis: a systematic review and metaanalysis. JACC Cardiovasc Imaging. 10(4):411-20. 10.1016/j.jcmg.2016.05.009.

10. Puntmann VO, Isted A, Hinojar R, Foote L, Carr-White G, Nagel E. T1 and T2 mapping in recognition of early cardiac involvement in systemic sarcoidosis. Radiology. 2017;285:63-72. [PubMed: 28448233]

11. Kim SJ, Pak K, Kim K. Diagnostic performance of F-18 FDG PET for detection of cardiac sarcoidosis; a systematic review and meta-analysis. J Nucl Cardiol. 2019 10.1007/ s12350-018-01582-y.

12. Blankstein R, Osborne M, Naya M, et al. Cardiac positron emission tomography enhances prognostic assessments of patients with suspected cardiac sarcoidosis. J Am Coll Cardiol. 2014;63:329-36. [PubMed: 24140661]

13. Sperry BW, Tamarappoo BK, Oldan JD, Javed O, Culver DA, Brunken R, et al. Prognostic impact of extent, severity, and heterogeneity of abnormalities on 18F-FDG PET scans for suspected cardiac sarcoidosis. JACC Cardiovasc Imaging. 2018;11:336-45. [PubMed: 28823747]

14. Vita T, Okada DR, Veillet-Chowdhury M, et al. Complementary value of cardiac magnetic resonance imaging and positron emission tomography/computed tomography in the assessment of cardiac sarcoidosis. Circ Cardiovasc Imaging. 2018;11:e007030. [PubMed: 29335272]

15. Slart RHJA, Glaudemans AWJM, Lancellotti P, et al. A joint procedural position statement on imaging in cardiac sarcoidosis: from the Cardiovascular and Inflammation \& Infection Committees of the European Association of Nuclear Medicine, the European Association of Cardiovascular Imaging, and the American Society of Nuclear Cardiology. Eur Heart J Cardiovasc Imaging. 2017;18: 1073-89. [PubMed: 28984894]

16. Wicks EC, Menezes LJ, Barnes A, et al. Diagnostic accuracy and prognostic value of simultaneous hybrid 18 F-fluorodeoxyglucose positron emission tomography/magnetic resonance imaging in cardiac sarcoidosis. Eur Heart J Cardiovasc Imaging. 2018;19:757-67. [PubMed: 29319785]

17. Kiko T, Yoshihisa A, Kanno Y, et al. A multiple biomarker approach in patients with cardiac sarcoidosis. Int Heart J. 2018;59: 996-1001. [PubMed: 30101857]

18. Fujiwara W, Kato Y, Hayashi M, et al. Serum microRNA-126 and - 223 as new-generation biomarkers for sarcoidosis in patients with heart failure. J Cardiol. 2018;72:452-7. [PubMed: 30054123]

19. Mankad P, Syed H, Syed A et al. C-reactive protein is elevated in sarcoidosis patients with ventricular tachycardia and heart failure. Hear. Rhythm Soc 2019 Abstr.

20. Liang JJ, Hebl VB, DeSimone CV, et al. Electrogram guidance: a method to increase the precision and diagnosticyieldofendomyocardial biopsy for suspectedcardiac sarcoidosisand myocarditis. JACC Hear Fail. 2014;2:466-73.

21. Segawa M, Fukuda K, Nakano M, Kondo M, Satake H, Hirano M, et al. Time course and factors correlating with ventricular tachyarrhythmias after introduction of steroid therapy in cardiac sarcoidosis. Circ Arrhythm Electrophysiol. 2016;9:1-9. 
22. Nagai S, Yokomatsu T, Tanizawa K, Ikezoe K, Handa T, Ito Y, et al. Treatment with methotrexate and low-dose corticosteroids in sarcoidosis patients with cardiac lesions. Intern Med. 2014;53:427-33. [PubMed: 24583430]

23. Birnie DH, Nery PB, Ha AC, Beanlands RSB. Cardiac Sarcoidosis. J Am Coll Cardiol. 2016;68:411-21. [PubMed: 27443438]

24. Kron J, Chicos A, Bogun F et al. (2017) Treatment of cardiac sarcoidosis with steroids and steroidsparing immunosuppressants: findings from the Cardiac Sarcoidosis Consortium. Hear. Rhythm Soc 2017 Abstr.

25. Sadek MM, Yung D, Birnie DH, Beanlands RS, Nery PB. Corticosteroid therapy for cardiac sarcoidosis: a systematic review. Can J Cardiol. 2013;29:1034-41. [PubMed: 23623644]

26. Okada DR, Smith J, Derakhshan A, Gowani Z, Misra S, Berger RD, et al. Ventricular arrhythmias in cardiac sarcoidosis. Circulation. 2018;138:1253-64. [PubMed: 30354431]

27. Papageorgiou N, Providência R, Bronis K, Dechering DG, Srinivasan N, Eckardt L, et al. Catheter ablation for ventricular tachycardia in patients with cardiac sarcoidosis: a systematic review. Europace. 2018;20:682-91. [PubMed: 28444174]

28. Al-Khatib SM, Stevenson WG, Ackerman MJ, et al. 2017 AHA/ACC/HRS guideline for management of patients with ventricular arrhythmias and the prevention of sudden cardiac death: a report of the American College of Cardiology/American Heart Association Task Force on Clinical Practice Guidelines and the Heart Rhythm Society. Heart Rhythm. 2018;15:e73-e189. [PubMed: 29097319]

29. Naruse Y, Sekiguchi Y, Nogami A, et al. Systematic treatment approach to ventricular tachycardia in cardiac sarcoidosis. Circ Arrhythm Electrophysiol. 2014;7:407-13. [PubMed: 24837644]

30. Muser D, Santangeli P, Pathak RK, et al. Long-term outcomes of catheter ablation of ventricular tachycardia in patients with cardiac sarcoidosis. Circ Arrhythm Electrophysiol. 2016;9:1-13.

31•. Yalagudri S, Zin Thu N, Devidutta S, Saggu D, Thachil A, Chennapragada S, et al. Tailored approach for management of ventricular tachycardia in cardiac sarcoidosis. J Cardiovasc Electrophysiol. 2017;28:893-902 [PubMed: 28429512] Characterization of underlying disease in cardiac sarcoidosis via PET-CT and tailoring management of VT accordingly can help improve clinical outcomes.

32. Kron J, Sauer W, Schuller J, et al. Efficacy and safety of implantable cardiac defibrillators for treatment of ventricular arrhythmias in patients with cardiac sarcoidosis. EP Eur. 2013;15:347-54.

33. Schuller JL, Zipse M, Crawford T, et al. Implantable cardioverter defibrillator therapy in patients with cardiac sarcoidosis. J Cardiovasc Electrophysiol. 2012;23:925-9. [PubMed: 22812589]

34. Betensky BP, Tschabrunn CM, Zado ES, Goldberg LR, Marchlinski FE, Garcia FC, et al. Longterm follow-up of patients with cardiac sarcoidosis and implantable cardioverter-defibrillators. Heart Rhythm. 2012;9:884-91. [PubMed: 22338670]

35. Nordenswan HK, Lehtonen J, Ekström K, et al. Outcome of cardiac sarcoidosis presenting with high-grade atrioventricular block. Circ Arrhythm Electrophysiol. 2018;11:e006145 [PubMed: 30354309] Findings from this study provide further support for the Heart Rhythm Expert Consensus recommendation to implant intracardiac cardioverter defibrillator for cardiac sarcoidosis patient when permanent pacing is needed.

36. Kusumoto FM, Schoenfeld MH, Barrett C, et al. 2018 ACC/AHA/HRS guideline on the evaluation and management of patients with bradycardia and cardiac conduction delay. Circulation. 2019 10.1161/cir.0000000000000628.

37. Aizer A, Stern EH, Gomes JA, Teirstein AS, Eckart RE, Mehta D. Usefulness of programmed ventricular stimulation in predicting future arrhythmic events in patients with cardiac sarcoidosis. Am J Cardiol. 2005;96:276-82. [PubMed: 16018857]

38. Zipse M, Tzou W, Schuller J et al. (2019) Electrophysiologic testing for diagnostic evaluation and risk stratification in patients with suspected cardiac sarcoidosis with preserved left and right ventricular systolic function. J Cardiovasc Electrophysiol In Press:

39. Smedema JP, van Geuns RJ, Ector J, Heidbuchel H, Ainslie G, Crijns HJGM. Right ventricular involvement and the extent of left ventricular enhancement with magnetic resonance predict adverse outcome in pulmonary sarcoidosis. ESC Hear Fail. 2018;5:157-71. 
40. Juneau D, Nery P, Russo J, de Kemp RA, Leung E, Beanlands RSB, et al. How common is isolated cardiac sarcoidosis? Extra-cardiac and cardiac findings on clinical examination and whole-body 18F-fluorodeoxyglucose positron emission tomography. Int J Cardiol. 2018;253:189-93. [PubMed: 29306462]

41. Adamson P, Melton I, O’Donnell J, MacDonald S, Crozier I. Cardiac sarcoidosis: the Christchurch experience. Intern Med J. 2014;44:70-6. [PubMed: 24450522]

42. Judson MA. Screening sarcoidosis patients for cardiac sarcoidosis: what the data really show. Respir Med. 2019;154:155-7. [PubMed: 31126731] 


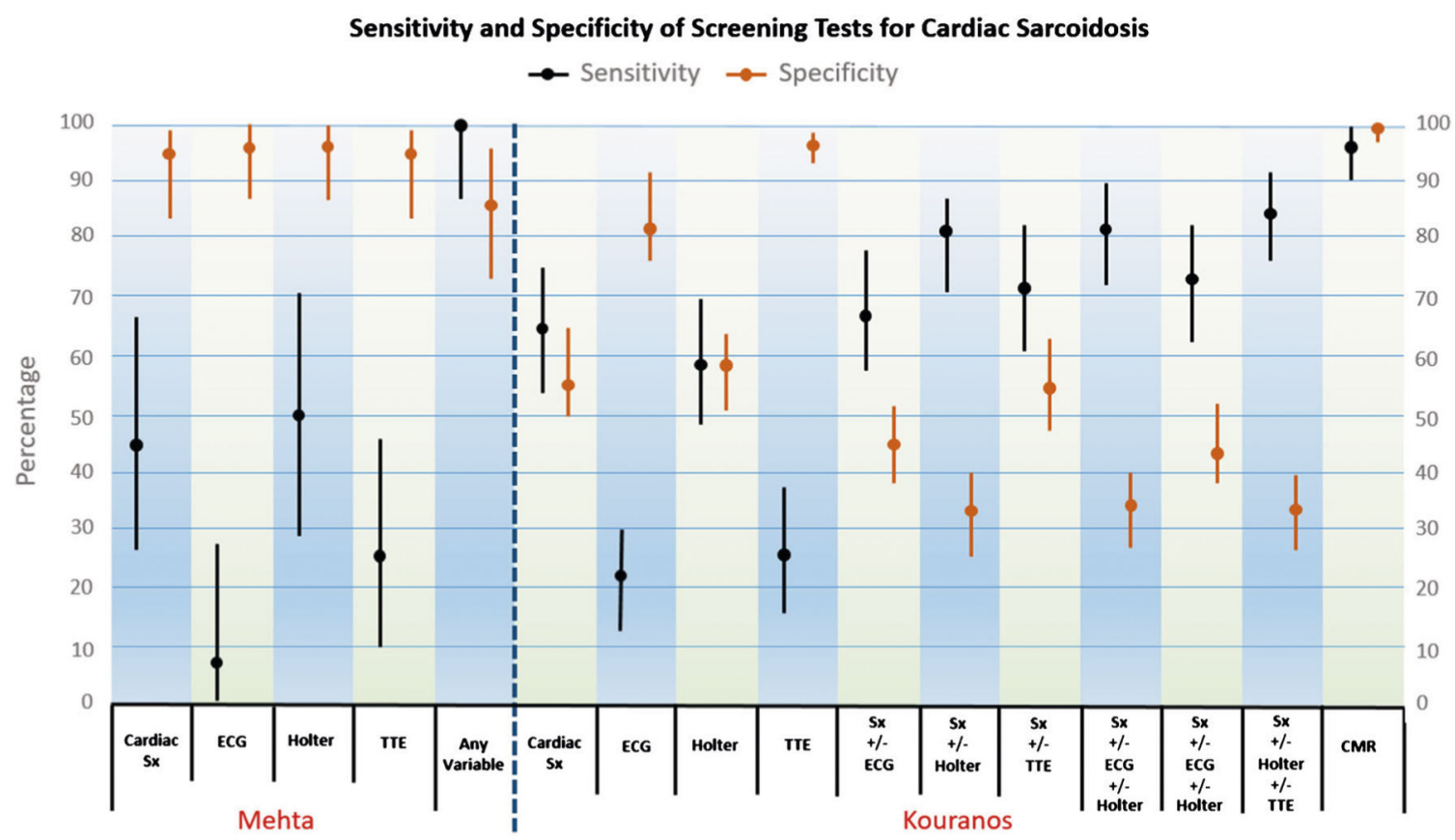

Fig. 1.

The sensitivity and specificity of various tests for the diagnosis of cardiac sarcoidosis in two sarcoidosis cohorts. Mehta: A sarcoidosis cohort of 62 patients from New York City, USA. Kouranos: A sarcoidosis cohort of 321 patients from Athens, Greece. Orange lines: 95\% confidence intervals for specificity of various tests with the means identified; Black lines: $95 \%$ confidence intervals for sensitivity of various tests with the means identified. Cardiac sx, cardiac symptoms; ECG, electrocardiogram; Holter, 24-h ambulatory electrocardiographic monitoring; TTE, transthoracic echocardiogram; any variable, combination of all previous tests; CMR, cardiac nuclear magnetic imaging. (Reproduced from: Judson MA. Respir Med 2019, 154:155-157, with permission from Elsevier) [42] 
a Short axis view

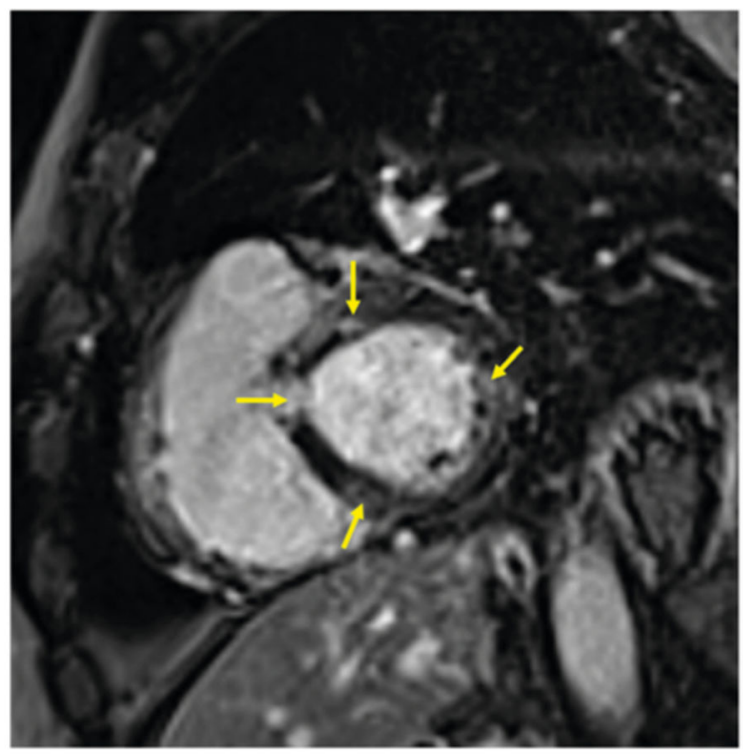

\section{b 4 chamber view}

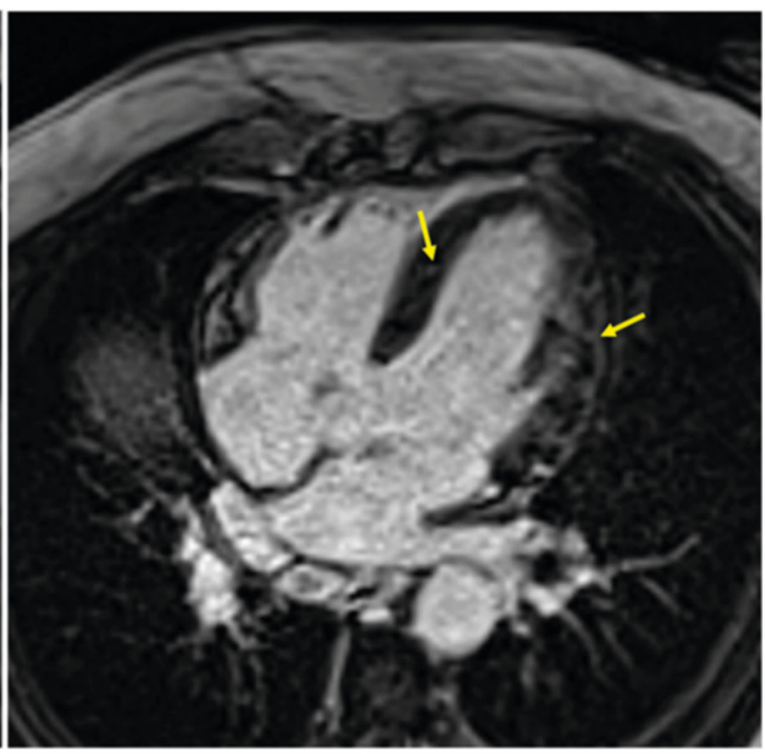

Fig. 2.

Cardiac magnetic resonance image of a 61-year-old male with cardiac sarcoidosis showing patchy late gadolinium enhancement (yellow arrows) involving the base of the anteroseptum, and multiple areas of the anteroseptum and inferoseptum and lateral wall shown in the short axis (a) and 4 chamber view (b) consistent with cardiac sarcoidosis 
a Before methotrexate

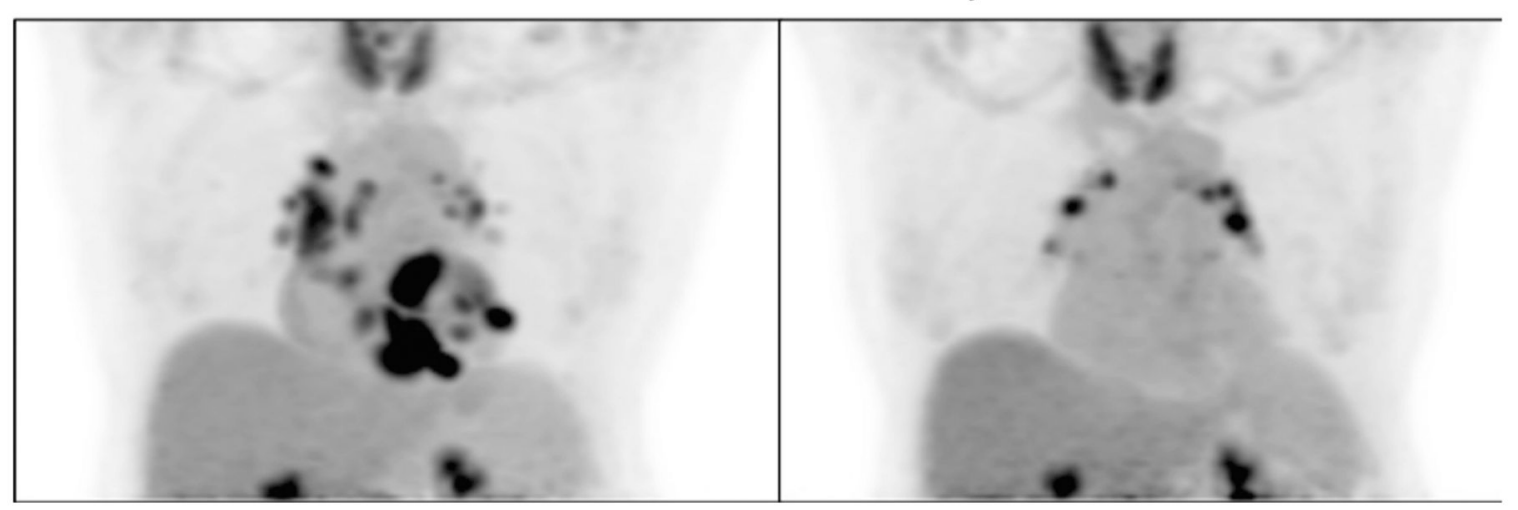

Fig. 3.

FDG-PET of a 63-year-old female on methotrexate for cardiac sarcoidosis before (a) and during (b) therapy with methotrexate for 2 years, exhibiting a significant decrease in FDG uptake in the heart suggesting adequate response to therapy. The scan continues to show FDG uptake in mediastinal lymph nodes 
Mankad et al.

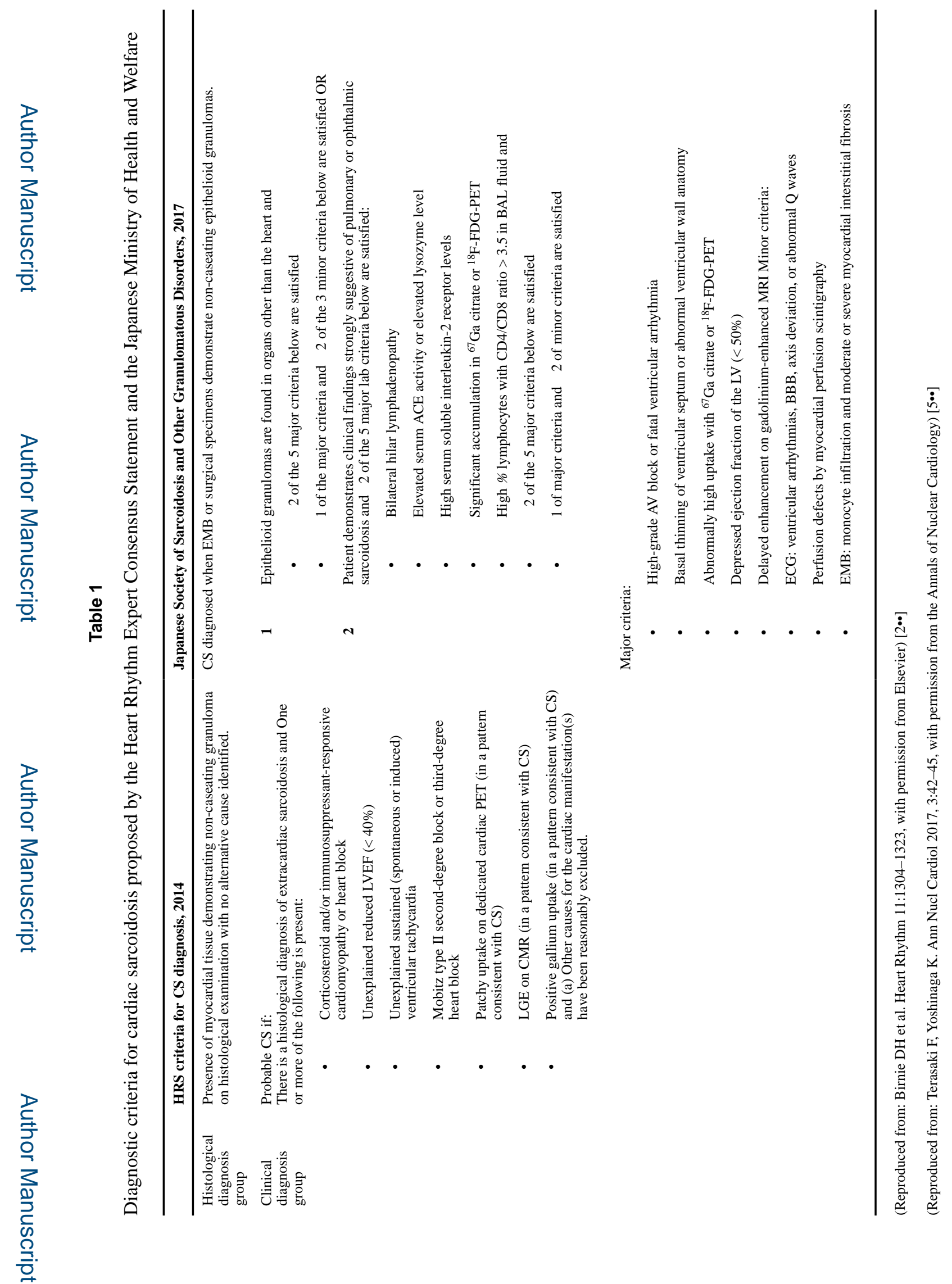

Curr Cardiol Rep. Author manuscript; available in PMC 2020 March 24. 